\title{
Cantharidin inhibits melanoma cell proliferation via the miR-21-mediated PTEN pathway
}

\author{
ZHEN MU ${ }^{1,2}$ and QING SUN ${ }^{1}$ \\ ${ }^{1}$ Department of Dermatology, Qilu Hospital of Shandong University, Jinan, Shandong 250012; \\ ${ }^{2}$ Department of Dermatology, Affiliated Hospital of Taishan Medical University, Taian, Shandong 271000, P.R. China
}

Received February 20, 2018; Accepted July 26, 2018

DOI: $10.3892 / \mathrm{mmr} .2018 .9440$

\begin{abstract}
Cantharidin (CTD) is an active component isolated from the blister beetle that has been demonstrated to exert antitumor effects on multiple types of cancer. The current study aimed to investigate whether the potential inhibitory effects of CTD exist in human melanoma cells and to assess the underlying antitumor mechanisms of CTD. Using the Cell Counting Kit- 8 assay, it was demonstrated that CTD treatment reduced A375 cell proliferation significantly in a dose-dependent manner. The colony formation assay demonstrated that CTD treatment could decrease the number of A375 cell colonies. Using subcutaneous xenograft tumor models, it was also demonstrated that CTD retarded solid tumor growth significantly. Furthermore, CTD treatment could induce A375 cell apoptosis, as detected by Annexin V-fluorescein isothiocyanate/propidium iodide staining and western blot analysis. Notably, CTD treatment reduced microRNA (miR)-21 expression and enhanced phosphatase and tensin homolog (PTEN) protein expression levels in A375 cells. Furthermore, overexpressing miR-21 in A375 cells with the miR-21 agomir blocked the antitumor effect of CTD both in vitro and in vivo. Finally, it was demonstrated that the inhibitory effects of CTD on A375 cells may be regulated by attenuating miR-21-mediated PTEN suppression. Based on these observations, it was suggested that CTD be used as a novel anti-proliferation agent of human melanoma via targeting the miR-21-PTEN signaling pathway.
\end{abstract}

\section{Introduction}

Melanoma, as a cutaneous cancer caused by the malignant transformation of melanocytes, is the most aggressive form and common cause of skin cancer-associated mortality in humans $(1,2)$. Risk factors for melanoma include age, sex, race,

Correspondence to: Professor Qing Sun, Department of Dermatology, Qilu Hospital of Shandong University, 107 Wenhua Xi Road, Jinan, Shandong 250012, P.R. China

E-mail: sunqsd66@163.com

Key words: cantharidin, miR-21, PTEN, proliferation, melanoma, apoptosis the constitutive color of the skin and the geographical zone (1). Recently, the incidence of melanoma has demonstrated rapid growth worldwide (3). Surgery, radiotherapy and chemotherapies are common therapies for early-stage, non-metastasized melanoma, with most cases of melanoma being curable. However, once metastasized, the efficiency of treatment was reduced significantly (2). Chemotherapy is an extremely ineffective and unsatisfactory method for treating malignant melanoma because of drug resistance, which is characteristic of this disease and limits its usage (4). The development of novel agents for melanoma treatment in the clinic is urgently needed.

Cantharidin (CTD), a terpenoid that is isolated from Chinese blister beetles, has been used as a traditional Chinese medicine to treat tumors for a very long time (5). Until now, cantharidin and its derivatives have been proven to possess anticancer activities in various types of cancer, including pancreatic cancer (6), hepatoma (7), and glioma (8). Furthermore, CTD has been reported to suppress A375.S2 human melanoma cell migration, invasion and apoptosis through cell cycle arrest and the induction of apoptosis $(9,10)$. However, there is no available information demonstrating that CTD can inhibit the proliferation of melanoma and clarifying its underlying mechanisms.

MicroRNAs (miRs) are short (approximately $22 \mathrm{nt}$ ), endogenous non-coding RNAs that regulate gene expression by binding to the 3'-untranslated region (3'UTRs) of target genes, suppressing mRNA translation or degrading mRNA. Emerging evidence has demonstrated that miRs are involved in diverse biological processes and disease development, including a number of types of cancer. Recently, dysregulation of miR-21 was reported in melanoma $(11,12)$ and selective expression alteration of miR-21 has been one of the important mechanisms for melanoma treatment with high-intensity focused ultrasound (13). However, whether the roles of CTD in melanoma are associated with miR-21 remains unknown.

The present study aimed to investigate the role of CTD in regulating the proliferation of human melanoma A375 cells and its association with the miR-21-PTEN signaling pathway. It was demonstrated that in A375 cells CTD could inhibit proliferation and tumorigenesis, as well as induce apoptosis, possibly mediated by downregulating the expression of miR-21 and upregulating the expression of phosphatase and tensin homolog (PTEN). 


\section{Materials and methods}

Chemicals and reagents. CTD and dimethyl sulfoxide (DMSO) were purchased from Sigma-Aldrich; Merck KGaA (Darmstadt, Germany). RPMI-1640 medium (Thermo Fisher Scientific, Inc., Waltham, MA, USA), fetal calf serum (FCS) and L-glutamine were purchased from Gibco (Thermo Fisher Scientific, Inc.). Primary antibodies against PTEN, protein kinase B (AKT), protein kinase B (p) AKT, Bax, B-cell lymphoma-2 (bcl-2), caspase-3, anti-GAPDH and the appropriate secondary antibodies were purchased from ProteinTech Group, Inc. (Chicago, IL, USA). The enhanced chemiluminescence (ECL) detection system was obtained from Millipore (EMD Millipore, Billerica, MA, USA).

Cell culture. The A375 human melanoma cancer cell line was purchased from the American Type Culture Collection (Manassas, VA, USA). Cells were cultured at $37^{\circ} \mathrm{C}$ in a $5 \% \mathrm{CO}_{2}$ humidified incubator in RPMI-1640 medium supplemented with $10 \%$ heat-inactivated FCS, $2 \mathrm{mM}$ glutamine, $100 \mathrm{U} / \mathrm{ml}$ penicillin and $0.1 \mathrm{mg} / \mathrm{ml}$ streptomycin.

Transfection of miR-21 agomir, antagomir, PTEN small interfering siRNA or overexpressed PTEN. Micron ${ }^{\mathrm{TM}}$ hsa-miR-21-5p agomir (5'-UAGCUUAUCAGACUGAUGUUG A-3') or agomir control (sequence unavailable), micrOFF ${ }^{\mathrm{TM}}$ hsa-miR-21-5p antagomir (5'-UCAACAUCAGUCUGAUAA GCUA-3') or antagomir control (sequence unavailable) were obtained from Guangzhou RiboBio Co., Ltd., (Guangzhou, China; $10 \mathrm{nM}$ ). SignalSilence ${ }^{\circledR}$ PTEN siRNA (5'-GCATAT GGAAAGCTTCATT-3') and its negative control oligonucleotides (5'-ACACGTCCGAACATACTAC-3') were purchased from Cell Signaling Technology $(50 \mathrm{nM})$. Transfections were performed using Lipofectamine ${ }^{\mathrm{TM}} 2000$ (Invitrogen; Thermo Fisher Scientific, Inc.) according to the manufacturer's protocol. For overexpression, the full-length PTEN sequence was cloned into the lentiviral vector [Obio Technology (Shanghai) Corp., Ltd., Shanghai, China]. Following amplification, lentiviral-PTEN (LV-PTEN) or empty vector (Vector) as a control was transduced into A375 cells at a concentration of $5 \times 10^{4}$ transducing units/ml using polybrene [Obio Technology (Shanghai) Corp.] according to the manufacturer's protocol. Next, $5 \times 10^{5}$ cells were seeded into 6-well plates. A total of $48 \mathrm{~h}$ following transfection, the cells were harvested for subsequent experiments.

Reverse transcription-quantitative polymerase chain reaction $(R T-q P C R)$. Total RNA was isolated from the A375 cell line using Trizol (Invitrogen; Thermo Fisher Scientific, Inc.). The concentration and purity of these RNA samples were detected. The total RNA samples were reverse transcribed into complementary DNA (cDNA) using PrimeScript RT Reagent kit (Takara Biotechnology Co., Ltd., Dalian, China). qPCR was performed using the MyiQ Real-Time PCR Detection System (Bio-Rad Laboratories, Inc., Hercules, CA, USA) and the SYBR-Green I premix (Takara Biotechnology Co., Ltd.). U6 or GAPDH mRNA were used as an endogenous control. The thermocycling conditions were as follows: $95^{\circ} \mathrm{C}$ for $10 \mathrm{~min}$, 40 cycles of $95^{\circ} \mathrm{C}$ for $15 \mathrm{sec}$ and $60^{\circ} \mathrm{C}$ for $1 \mathrm{~min}$. The experiment was repeated three times and the levels of miR were normalized for each well to the levels of U6 using the $2^{-\Delta \Delta C q}$ method (14). The forward (F) and reverse (R) primers used in this study were as follows: miR-21, F: 5'-GGACTAGCTTAT CAGACTG-3' and R: 5'-CATCAGATGCGTTGCGTA-3'; U6 F: 5'-ATTGGAACGATACAGAGAAGAT-3' and R, 5'-GGA ACGCTTCACGAATTT-3'; PTEN F: 5'-CGGCAGCATCAA ATGTTTCAG-3' and R: 5'-AACTGGCAGGTAGAAGGC AACTC-3'.

Western blot analysis. Cells were collected and homogenized in ice-cold RIPA buffer (Beyotime Institute of Biotechnology, Shanghai, China). The amounts of protein from each treatment were detected and normalized using a bicinchoninic acid assay. Proteins $(20 \mu \mathrm{g} / \mathrm{lane})$ were separated by $10 \%$ SDS-PAGE and were subsequently transferred to polyvinylidene difluoride membranes. The membranes were blocked with 5\% non-fat milk at room temperature for $2 \mathrm{~h}$ and then separately probed overnight at $4{ }^{\circ} \mathrm{C}$ with the following primary antibodies: Anti-GAPDH (1:5,000; cat. no. 10494-1-AP), Bcl-2 (1:1,000; cat. no. 12789-1-AP), Bax (1:1,000; cat. no. 50599-2-Ig), caspase-3 (1:1,000, cat. no. 19677-1-AP), PTEN (1:1,000, cat. no. 22034-1-AP), AKT (1:1,000, cat. no. 10176-2-AP), p AKT (1:1,000; cat. no. 66444-1-Ig). After washing with TBST buffer (0.1\% Tween-20), the membranes were incubated with horseradish peroxidase-conjugated goat anti-mouse immunoglobulin $(\mathrm{Ig}) \mathrm{G}$ or goat anti-rabbit $\operatorname{IgG}(1: 5,000$; cat. no. SA00001-1 or SA00001-2) for $1 \mathrm{~h}$ at room temperature. Images were then captured of the membranes using the ECL detection system (EMD Millipore). Densitometry analysis of the bands was performed using Quantity One (version 4.5.0; Bio-Rad Laboratories, Inc.). The proteins were quantified and expressed as their ratio to GAPDH.

Cell Counting Kit-8 (CCK-8) assay. A375 cells were seeded in 96-well plates at a density of 1,000 cells/well in $100 \mu \mathrm{l}$ culture medium with different doses $(0,0.2,1$ or $5 \mu \mathrm{M})$ of CTD (dissolved with $0.5 \%$ dimethyl sulfoxide; Sigma-Aldrich; Merck KGaA, Darmstadt, Germany) treatments. Next, at 24, 48, 72 and $96 \mathrm{~h}$ following treatment, CCK-8 solution was added to each well. The optical density of the cells was measured using a microplate reader (Bio-Rad Laboratories, Inc.) at $450 \mathrm{~nm}$.

Colony-formation assay. A375 cells were seeded in a 6-well plate $(600$ cells/well) in complete medium and were allowed to grow for $24 \mathrm{~h}$. The cells were then exposed to $5 \mu \mathrm{M}$ CTD or vehicle for $48 \mathrm{~h}$. After the drug was removed, the cells were washed with PBS and incubated for another 14 days in complete medium. The cells were stained with $1 \%$ crystal violet solution at room temperature. Following 10 min incubation, the excess crystal violet was washed out and the stained colonies were counted under an inverted microscope (Nikon Corporation, Tokyo, Japan).

Establishment of subcutaneous xenograft tumor models. Male BALB/C Nu mice were obtained from Vital River Laboratory Animal Technology Co., Ltd., (Beijing, China) at 3- to 4-weeks old and weighing 18-20 g. Five mice per cage were housed together under a 12/12 h light/dark cycle in standard laboratory specific pathogen free cages $\left(22 \pm 2^{\circ} \mathrm{C}, 50-60 \%\right.$ humidity), 
with food and water ad libitum. A375 cells were digested using $0.25 \%$ pancreatin and were counted. The cell concentration was adjusted to $1 \times 10^{7}$ cells $/ \mathrm{ml}$ and a $0.1 \mathrm{ml}$ cell suspension was subcutaneously injected into the right armpit of the mice. The volume of tumors was measured with a caliper every 3 days using the following equation: Tumor volume=width $(\mathrm{W})^{2}$ xlength $(\mathrm{L}) / 2$. When the tumor volumes reached approximately $100 \mathrm{~mm}^{3}$, the mice were randomly and equally divided into 2 groups and were treated with control or CTD (intraperitoneal injected with $200 \mu \mathrm{l}$ vehicle or CTD, $0.5 \mathrm{mg} / \mathrm{kg} /$ day) for 3 weeks. In addition, certain mice in the CTD-treated group were intratumorally injected with miR21 agomir (10 nM diluted with $50 \mu \mathrm{l}$ of PBS, three times per week; Guangzhou Ribobio Co., Ltd.) or agomir control accompanied with CTD treatment ( $n=6$ per group). A total of 30 mice were included in the present study. All the experiments were performed in accordance with the relevant guidelines and approved by the Institutional Animal Care and Use Committee (IACUC) of Taishan Medical University.

Flow cytometric analysis. Following treatment with CTD for $48 \mathrm{~h}$, the cells were harvested and reconstituted to $1-5 \times 10^{6} / \mathrm{ml}$. Next, $100 \mu \mathrm{l}$ cell suspension was transferred into a $5 \mathrm{ml}$ flow tube, followed by staining with $5 \mu \mathrm{l}$ of Annexin V/fluorescein isothiocyanate (FITC; Beijing 4A Biotech Co., Beijing, China) for $5 \mathrm{~min}$ at room temperature in the dark. Next, the cells were stained with $10 \mu \mathrm{l}$ propidium iodide (PI) and $400 \mu \mathrm{l}$ PBS, and then collected and detected by flow cytometry. The results were analyzed using Flowjo software (version 9.3.2; FlowJo LLC, Ashland, OR, USA).

Statistical analysis. Data analysis was performed using SPSS 18.0 software (SPSS, Inc., Chicago, IL, USA). All the data were presented as the mean \pm standard deviation. The data of only two groups were analyzed using a Student's t-test. One-way analysis of variance followed by the Student Newman Keuls test was used for the analysis of three or more groups. $\mathrm{P}<0.05$ was considered to indicate a statistically significance difference.

\section{Results}

CTD inhibits the proliferation and tumorigenesis of melanoma. The effects of different doses of CTD $(0.2,1$ and $5 \mu \mathrm{M}$ ) were first evaluated on the growth of A375 cells using the CCK-8 assay. The results demonstrated that the OD value of $5 \mu \mathrm{M}$ CTD-treated A375 cells was significantly decreased compared with the vehicle control at 48, 72 and $96 \mathrm{~h}(\mathrm{P}<0.05)$. Next, $1 \mu \mathrm{M}$ CTD significantly decreased the OD value of A375 cells only at $96 \mathrm{~h}(\mathrm{P}<0.01)$ and significantly increased compared with $5 \mu \mathrm{M}$ CTD $(\mathrm{P}<0.01)$. Additionally, $0.2 \mu \mathrm{M}$ CTD demonstrated no effect on the OD value (Fig. 1A). These results suggest that CTD had an inhibitory effect on the proliferation of A375 cells in a dose-dependent manner. Therefore, $5 \mu \mathrm{M}$ CTD was used in subsequent in vitro experiments. The colony formation assay was also used to detect the roles of CTD in the growth of A375 cells. The plates with $5 \mu \mathrm{M}$ CTD treatment exhibited significantly fewer cell clones compared with the control group $(\mathrm{P}<0.01$; Fig. $1 \mathrm{~B}$ and $\mathrm{C})$. All these results indicated that CTD inhibits the proliferation of
A375 cells effectively in vitro. Whether CTD had antitumor effects on A375 cell xenografts in vivo was next investigated. The results demonstrated that CTD retarded the growth of the xenograft tumor significantly and the final tumor volume was significantly smaller compared with the vehicle-treated group $(\mathrm{P}<0.01$; Fig. 1D and E), suggesting that CTD inhibited the tumorigenesis of melanoma in vivo.

CTD promotes the apoptosis of A375 cells. To observe the effect of CTD on cell apoptosis in A375 cells, an Annexin V-FITC/PI staining assay was conducted. As shown in Fig. 2A and B, CTD increased the number of apoptotic cells compared with those in the vehicle control group $(\mathrm{P}<0.01)$. These data suggest that CTD may promote human melanoma cell apoptosis. To study further the effects of CTD on cell apoptosis western blot analysis was performed. The expression level of Bcl-2, an anti-apoptosis protein, was significantly decreased following CTD treatment $(\mathrm{P}<0.01)$, while the expression levels of pro-apoptosis proteins Bax and active caspase- 3 were significantly increased $(\mathrm{P}<0.01$; Fig. $2 \mathrm{C}$ and $\mathrm{D})$. These results indicate that CTD could effectively induce the apoptosis of A375 cells.

CTD suppresses miR-21 and increases PTEN expression in A375 cells. Next, alterations in the relative expression levels of miR-21 and its target gene PTEN following CTD administration were measured. A higher miR-21 expression in A375 cells was decreased significantly following CTD treatment $(\mathrm{P}<0.01$; Fig. 3A). Furthermore, PTEN, the potential downstream gene of miR-21, was upregulated in both the mRNA and protein expression levels following CTD treatment $(\mathrm{P}<0.01$; Fig. 3B and C). These results suggested that miR-21 and PTEN may be involved in the effects of CTD on A375 cells. The AKT kinase is the one of the important downstream effectors of PTEN. Accompanied with the increased expression of PTEN following CTD treatment, the expression of the active form of AKT, pAKT, was decreased (Fig. 3C). These results proved that CTD may suppress the AKT pathway by increasing PTEN expression.

Antitumor effects of CTD via attenuating miR-21-mediated PTEN suppression. To further verify the association between miR-21 downregulation and CTD-induced antitumor effects, miR-21 agomir and antagomir were used to observe the CTD effects on A375 proliferation and tumorigenesis. The results demonstrated that the miR-21 agomir or antagomir could significantly increase or decrease, respectively, the expression of miR-21 in A375 cells (both $\mathrm{P}<0.01$; Fig. 4A). Compared with the CTD+antagomir/agomir control, the miR-21 agomir $(\mathrm{P}<0.01)$ or antagomir $(\mathrm{P}<0.05)$ impeded or promoted the effect of CTD on the OD values, respectively (Fig. 4B). Furthermore, it was demonstrated that miR-21 agomir administration could significantly reverse the decrease in the xenograft tumor volumes caused by CTD treatment on the 21 st day $(\mathrm{P}<0.01$; Fig. $4 \mathrm{C})$. These results suggest that miR-21 is involved in the antitumor effect of CTD both in vitro and in vivo. Next, the roles of PTEN in the antitumor effect of CTD were evaluated. First, the effectiveness of the tools used to regulate PTEN expression was confirmed (Fig. 4D and E). Similarly, it was observed that PTEN overexpression or 

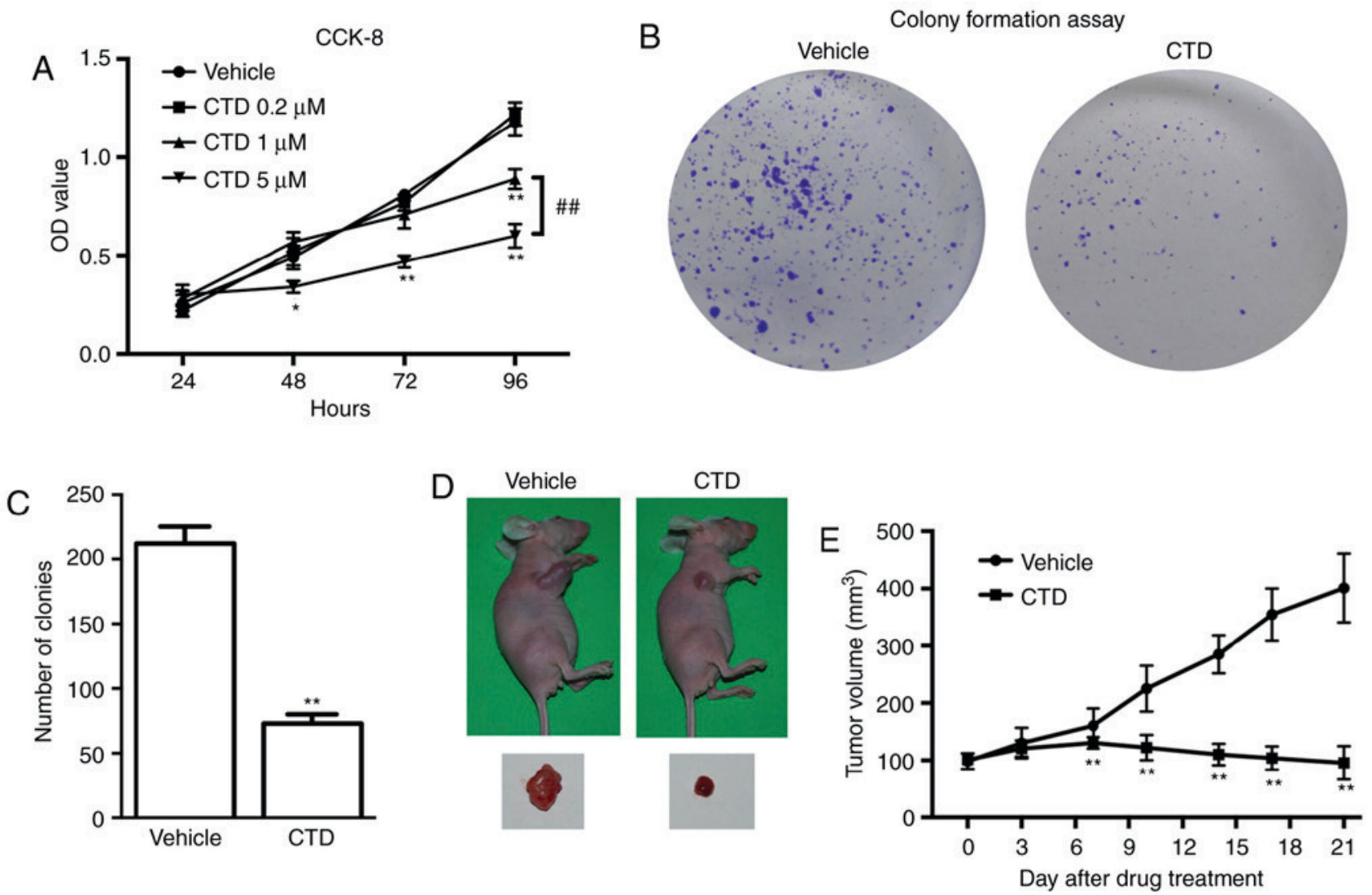

Figure 1. CTD inhibits the proliferation of A375 cells and tumorigenesis of melanoma. (A) Alterations in the viability of A375 cells following treatment with different doses of CTD as measured by a CCK- 8 assay. ${ }^{*} \mathrm{P}<0.05$ and ${ }^{* *} \mathrm{P}<0.01$ vs. the vehicle group at the indicated time points. ${ }^{\# \#} \mathrm{P}<0.01$ vs. the $1 \mu \mathrm{M}$ CTD group at $96 \mathrm{~h}$. The experiment was repeated three times. (B) Representative graphs of colony formation following CTD treatment. (C) Statistical analysis of the colony numbers. ${ }^{* * *} \mathrm{P}<0.01$ vs. the vehicle group. The experiment was repeated five times. (D) Representative graphs of in-site and isolated melanoma. (E) The tumor volume was recorded twice a week following CTD treatment for 3 weeks. ${ }^{* *} \mathrm{P}<0.01$ vs. the vehicle group at the indicated time points. $\mathrm{n}=6$ per group. All values are presented as the mean \pm standard deviation of the mean. CTD, Cantharidin; OD, optical density; CCK-8, Cell Counting Kit- 8 .

siRNA PTEN treatment could significantly enhance or impede the roles of CTD in A375 cell viability, respectively $(\mathrm{P}<0.01$; Fig. 4F). Finally, it was demonstrated that miR-21 agomir or antagomir administration blocked or enhanced the decreased effect of CTD on OD values, respectively, whereas a combination with PTEN overexpression or knockdown could, respectively, reverse these results (all $\mathrm{P}<0.05$; Fig. 4G). These results indicate that the antitumor effects of CTD occurred via attenuating miR-21-mediated PTEN suppression.

\section{Discussion}

In the present study, the effects and underlying mechanisms of CTD on melanoma proliferation were evaluated. CTD could effectively inhibit the viability and colony formation of A375 cells, decrease the size of xenograft tumors and induce A375 cell apoptosis, all of which were regulated via attenuating miR-21-mediated PTEN suppression.

Previous findings have shown that CTD exerts general anticancer effects on the proliferation and metastasis of a number of types of human cancer (5). It was also indicated that CTD inhibited human melanoma cell migration and invasion effectively (9). In addition to the inhibited roles in melanoma metastasis, in the present study, it was demonstrated that CTD could suppress the proliferation of melanoma both in vitro and in vivo. Furthermore, the melanoma cell apoptosis increased following CTD treatment, which may be associated with the inhibition of AKT signaling pathway. These results are in agreement with results of previous studies $(9,10)$ and indicates that CTD or its derivatives could be candidates for melanoma treatment.

However, because of the toxicity, CTD has a very narrow therapeutic window in clinical use $(5,15)$. In addition, CTD can accumulate in certain healthy organs and impair its normal physiological function (5). Therefore, to fully exploit CTD in the future, it is important to clarify the underlying mechanisms and identify selective downstream targets of CTD during cancer treatment. CTD has been demonstrated to be the inhibitor of protein phosphatases (PP) type 1 and type $2 \mathrm{~A}$ (PP2A), which serve key roles in regulating the signal transfer of the cell cycle, mitosis and apoptosis (16). However, the detailed underlying mechanisms of CTD remain unclear. Recently, miRs were demonstrated to serve important roles in cancer chemotherapy (17-19), but little is known about the involvement of miRs in CTD treatment. Abnormal miR-21 expression was demonstrated in melanoma tumor tissue samples and cell lines $(11,12,20)$. Furthermore, proliferation, invasion, migration and apoptosis are partly regulated by miR-21 in melanoma tumorigenesis $(13,20,21)$. Therefore, the selective inhibition of miR-21 is a promising therapy for melanoma. In the present study, it was demonstrated that the high expression of miR-21 in A375 cells was inhibited by 

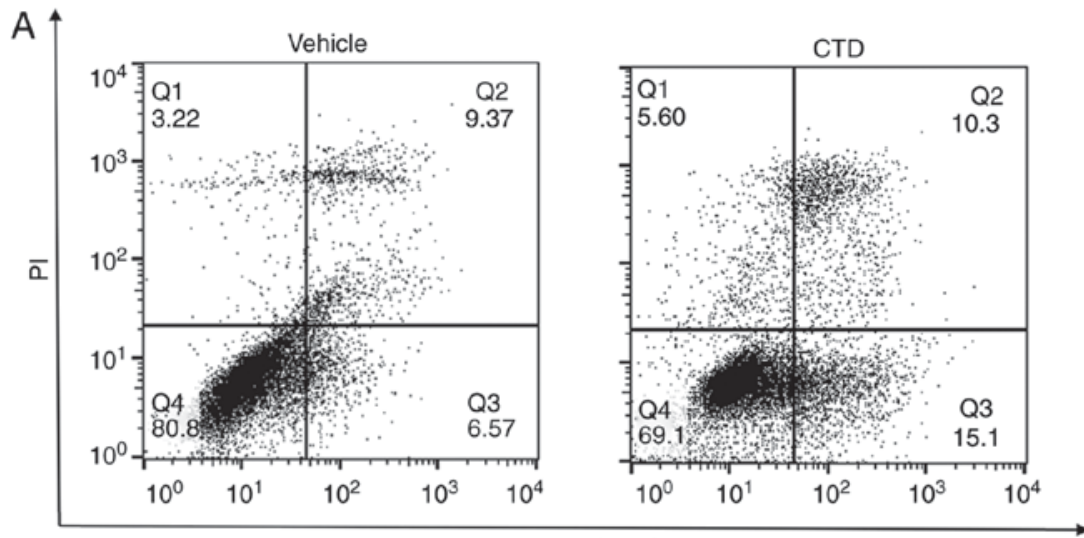

Annexin V-FITC
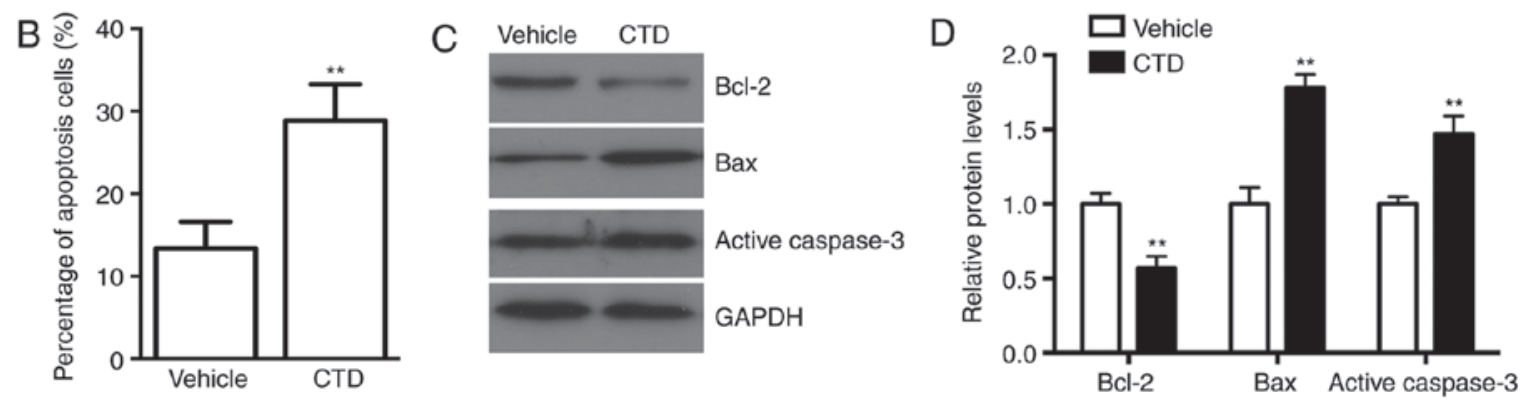

Figure 2. CTD induces the apoptosis of A375 cells. (A) Representative Annexin V-FITC/PI double-staining graphs. (B) Statistical analysis of the percentage of apoptotic cells. ${ }^{* *} \mathrm{P}<0.01$ vs. the vehicle group. The experiment was repeated five times. (C) Representative western blot graphs of Bcl-2, Bax and active caspase-3 following CTD treatment in A375 cells. (D) Statistical analysis of the gray value of blots. ${ }^{* *} \mathrm{P}<0.01$ vs. the vehicle group. The experiment was repeated five times. FITC, fluorescein isothiocyanate; Bcl-2, B-cell lymphoma-2; PI, propidium iodide.
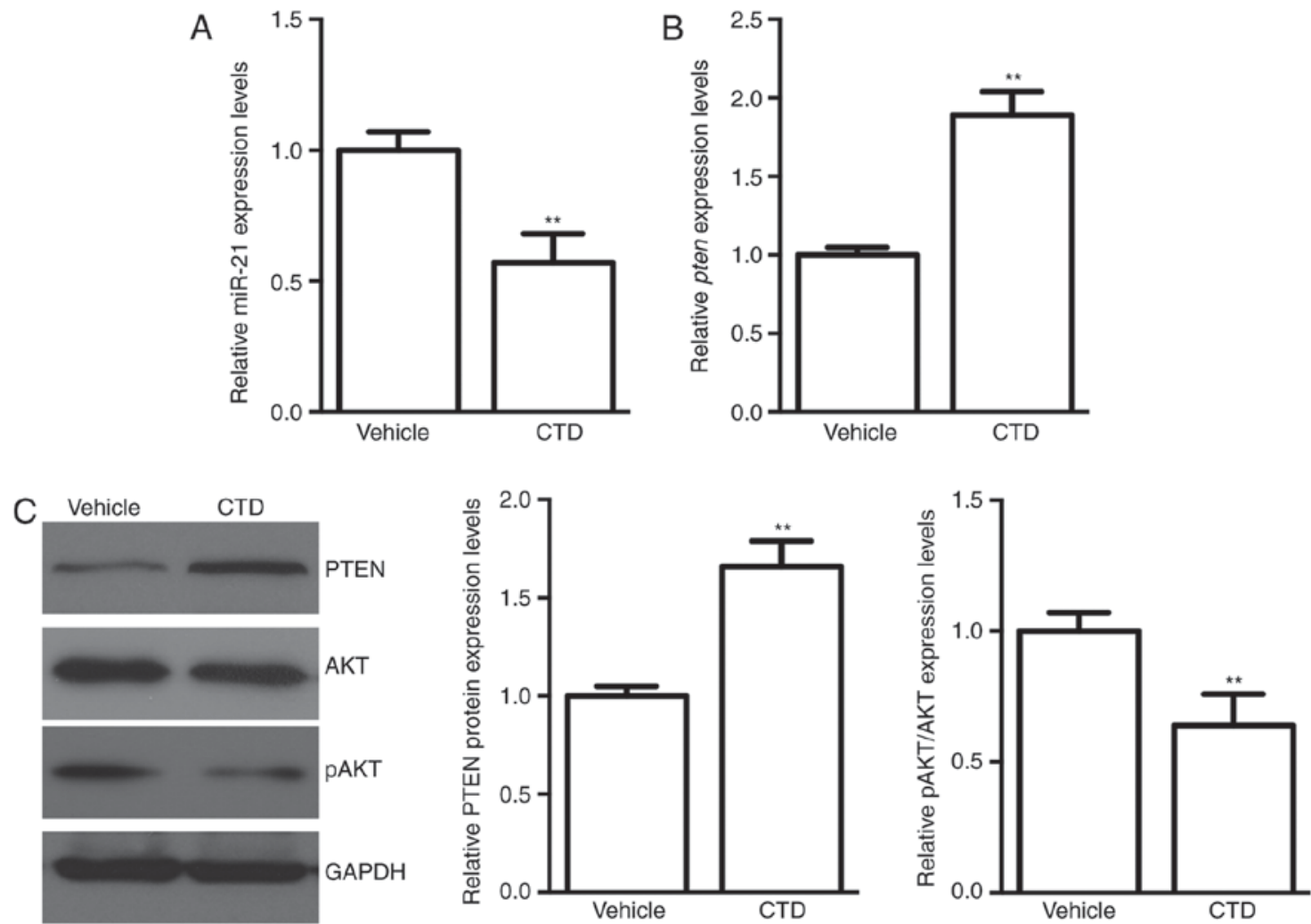

Figure 3. CTD inhibits the expression of miR-21 but increased the expression of PTEN in A375 cells. (A) Alterations in miR-21 expression following CTD treatment. ${ }^{* * *} \mathrm{P}<0.01$ vs. the vehicle group. The experiment was repeated five times. (B) Alterations in pten $\mathrm{mRNA}$ expression following CTD treatment. ${ }^{* *} \mathrm{P}<0.01$ vs. the vehicle group. $n=3$ per group. (C) The left panel demonstrated representative western blotting graphs of PTEN, AKT and pAKT following CTD treatment in A375 cells. The right panel demonstrates statistical analysis of the gray values of the blots. ${ }^{* *} \mathrm{P}<0.01$, vs. the vehicle group. The experiment was repeated five times. CTD, Cantharidin; pAKT, phosphorylated protein kinase B; PTEN, phosphatase and tensin homolog. 

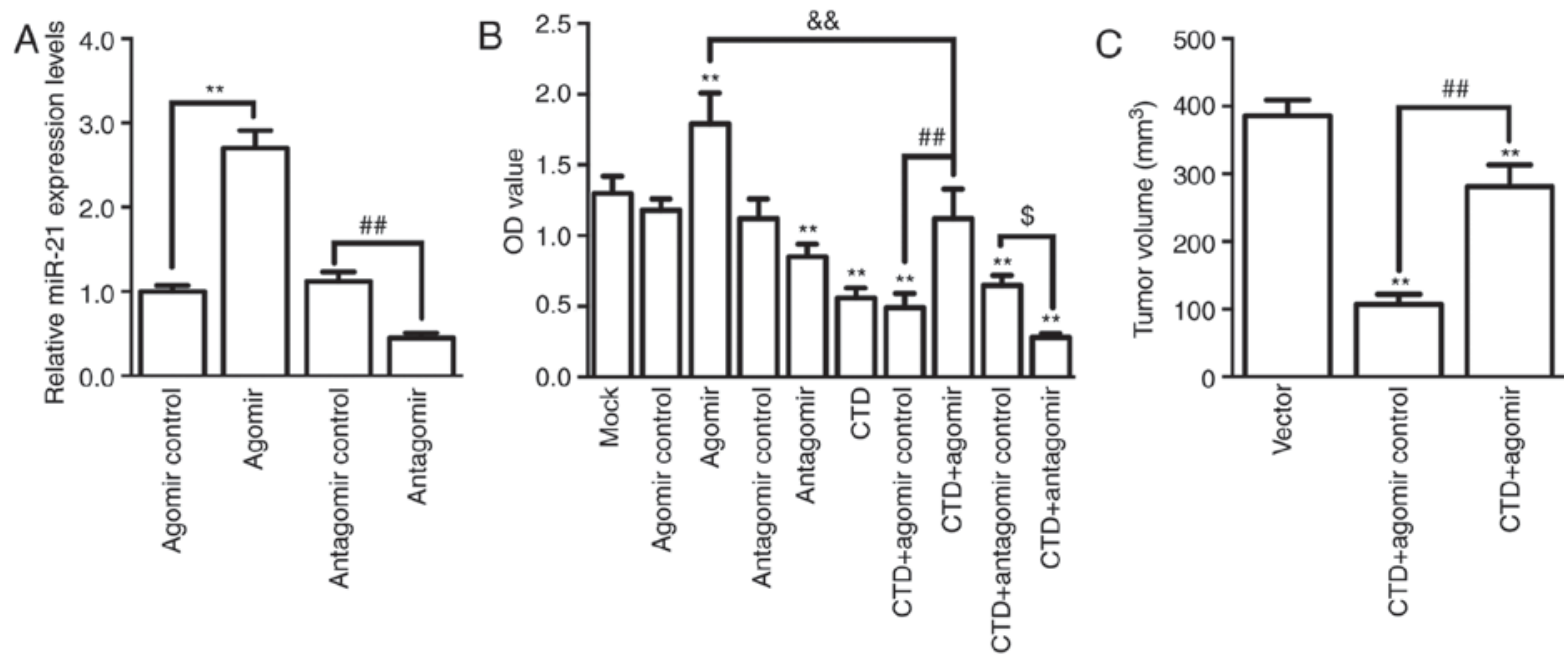


$\mathrm{E}$

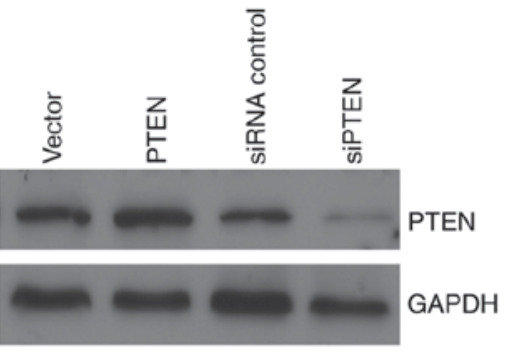

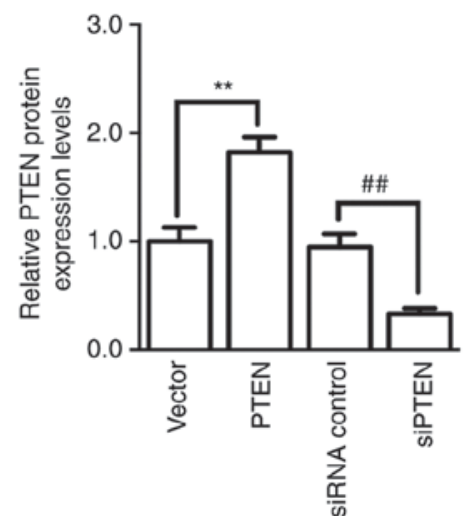

G

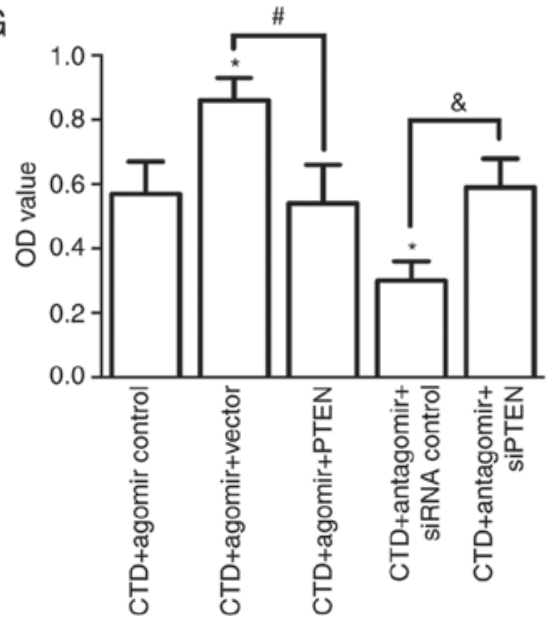

Figure 4. The antitumor roles of CTD in A375 melanoma cells are achieved by attenuating miR-21-mediated PTEN suppression. (A) Alterations in miR-21 expression following miR-21 agomir or antagomir treatment. ${ }^{* *} \mathrm{P}<0.01$; ${ }^{\# \#} \mathrm{P}<0.01$. The experiment was repeated three times. (B) Alterations in the viability of A375 cells following different treatments for $96 \mathrm{~h}$ as measured by the CCK-8 assay. ${ }^{* *} \mathrm{P}<0.01$ vs. the mock group. ${ }^{\# \#} \mathrm{P}<0.01 ;{ }^{\& \&} \mathrm{P}<0.01 ;{ }^{\$} \mathrm{P}<0.05$. The experiment was repeated three times. (C) Final tumor volumes following different treatments for 3 weeks. ${ }^{* *} \mathrm{P}<0.01$, compared with the vehicle group. ${ }^{\# \#} \mathrm{P}<0.01$. $\mathrm{n}=6$ per group. (D) Expression of pten in A375 cells following different treatments. LV-PTEN, overexpression of PTEN; siRNA PTEN, knockdown of PTEN by specific siRNA. ${ }^{* *} \mathrm{P}<0.01 ;{ }^{\# \#} \mathrm{P}<0.01$. The experiment was repeated three times. (E) The left panel presents representative western blot graphs of PTEN following different treatments in A375 cells. The right panel demonstrates statistical analysis of the gray values of the blots. ${ }^{* *} \mathrm{P}<0.01$; ${ }^{\# \#} \mathrm{P}<0.01$. The experiment was repeated five times. (F) Alterations in the viability of A375 cells following different treatments for $96 \mathrm{~h}$ as measured by the $\mathrm{CCK}-8$ assay. ${ }^{*} \mathrm{P}<0.05,{ }^{* *} \mathrm{P}<0.01$ vs. the mock group. ${ }^{\#} \mathrm{P}<0.05$; \&\& $<0.01$. The experiment was repeated three times. (G) Alterations in the viability of A375 cells following different treatments for $96 \mathrm{~h}$ as measured by the CCK-8 assay. ${ }^{*} \mathrm{P}<0.05$ vs. the CTD+agomir control group. ${ }^{*} \mathrm{P}<0.05 ;{ }^{\&} \mathrm{P}<0.05$. The experiment was repeated three times. PTEN, phosphatase and tensin homolog; siRNA, small interfering; CCK-8, Cell Counting Kit-8.

CTD. Overexpression or downregulation of miR-21 in A375 cells could impair or enhance the effect of CTD on cell proliferation, metastasis and apoptosis. These results suggest that
miR-21 was one of the important targets of CTD for melanoma treatment, a result that, to the best of our knowledge, has not been previously reported. 
miR-21 can regulate certain target genes and is involved in melanoma $(20,22)$. PTEN, a famous tumor suppressor, is one of the miR-21 target genes reported recently (23). Previous studies have demonstrated that PTEN can promote the host immune response against cancer cells by suppressing the intracellular levels of certain immunosuppressive factors, including interleukin (IL)-6, IL-10, vascular endothelial growth factor, and programmed cell death 1 ligand, by negatively regulating the AKT/protein kinase B signaling pathway (24-27). In addition, PTEN is associated with melanoma aggressiveness and a worse prognosis in patients (28). Recently, a number of studies have demonstrated that various drugs or compounds promote PTEN mRNA and protein expression to repress tumor formation and progression $(29,30)$. Therefore, whether the roles of CTD in A375 cells are associated with miR-21-regulated PTEN was further investigated in the present study. The putative relationship between the PTEN/AKT pathway and effects of CTD on A375 cell proliferation were investigated, which were mediated by miR-21. Certainly, miR-21 has other potential targets such as programmed cell death 4 and p53, in addition to PTEN $(23,31)$. Consequently, further studies are necessary to investigate whether other miR-21 target genes are involved in the CTD-induced repression of melanoma proliferation.

In conclusion, results of the present study provide evidence that CTD has anti-proliferative effects on human melanoma and underlying mechanisms are via miR-21 downregulation, increased PTEN expression, and decreased AKT activity. The identification of the miR-21/PTEN/AKT pathway in CTD treatment may be useful in the development of more efficacious and less toxic CTD analogs for melanoma chemotherapy in the future.

\section{Acknowledgements}

Not applicable.

\section{Funding}

This study was supported by the Projects of Medical and Health Technology Development Program in Shandong Province (grant no. 2017WS254).

\section{Availability of data and materials}

The datasets used and/or analyzed during the current study are available from the corresponding author on reasonable request.

\section{Authors' contributions}

ZM and QS designed the study. ZM performed all the experiments and analyzed the data. ZM and QS wrote and critically revised the manuscript.

\section{Ethics approval and consent to participate}

All experiments were performed in accordance with the relevant guidelines and approved by the Institutional Animal Care and Use Committee (IACUC) of Taishan Medical University.

\section{Patient consent for publication}

Not applicable.

\section{Competing interests}

The authors declare that they have no competing interests.

\section{References}

1. Apalla Z, Lallas A, Sotiriou E, Lazaridou E and Ioannides D: Epidemiological trends in skin cancer. Dermatol Pract Concept 7: $1-6,2017$.

2. Miller AJ and Mihm MC Jr: Melanoma. N Engl J Med 355: 51-65, 2006.

3. Siegel RL, Miller KD and Jemal A: Cancer Statistics, 2017. CA Cancer J Clin 67: 7-30, 2017.

4. Kalal BS, Upadhya D and Pai VR: Chemotherapy resistance mechanisms in advanced skin cancer. Oncol Rev 11: 326, 2017.

5. Deng LP, Dong J, Cai H and Wang W: Cantharidin as an antitumor agent: A retrospective review. Curr Med Chem 20: 159-166, 2013.

6. Shen M, Wu MY, Chen LP, Zhi Q, Gong FR, Chen K, Li DM, $\mathrm{Wu} \mathrm{Y}$, Tao $\mathrm{M}$ and $\mathrm{Li}$ W: Cantharidin represses invasion of pancreatic cancer cells through accelerated degradation of MMP2 mRNA. Sci Rep 5: 11836, 2015.

7. Zheng LH, Bao YL, Wu Y, Yu CL, Meng X and Li YX: Cantharidin reverses multidrug resistance of human hepatoma HepG2/ADM cells via down-regulation of P-glycoprotein expression. Cancer Lett 272: 102-109, 2008.

8. Xie D, Xie J, Wan Y, Ma L, Qi X, Wang K and Yang S: Norcantharidin blocks $W n t / \beta$-catenin signaling via promoter demethylation of WIF-1 in glioma. Oncol Rep 35: 2191-2197, 2016.

9. Ji BC, Hsiao YP, Tsai CH, Chang SJ, Hsu SC, Liu HC, Huang YP, Lien JC and Chung JG: Cantharidin impairs cell migration and invasion of A375.S2 human melanoma cells by suppressing MMP-2 and -9 through PI $3 \mathrm{~K} / \mathrm{NF}-\kappa \mathrm{B}$ signaling pathways. Anticancer Res 35: 729-738, 2015.

10. Hsiao YP, Tsai CH, Wu PP, Hsu SC, Liu HC, Huang YP, Yang JH and Chung JG: Cantharidin induces G2/M phase arrest by inhibition of $\mathrm{Cdc} 25 \mathrm{c}$ and Cyclin $\mathrm{A}$ and triggers apoptosis through reactive oxygen species and the mitochondria-dependent pathways of A375.S2 human melanoma cells. Int J Oncol 45: 2393-2402, 2014.

11. Wandler A, Riber-Hansen R, Hager H, Hamilton-Dutoit SJ, Schmidt $H$, Nielsen BS, Stougaard $M$ and Steiniche T: Quantification of microRNA-21 and microRNA-125b in melanoma tissue. Melanoma Res 27: 417-428. 2017.

12. Latchana N, Ganju A, Howard JH and Carson WE III: MicroRNA dysregulation in melanoma. Surg Oncol 25: 184-189, 2016.

13. Li H, Yuan SM, Yang M, Zha H, Li XR, Sun H, Duan L, Gu Y, Li AF, Weng YG, et al: High intensity focused ultrasound inhibits melanoma cell migration and metastasis through attenuating microRNA-21-mediated PTEN suppression. Oncotarget 7: 50450-50460, 2016.

14. Livak KJ and Schmittgen TD: Analysis of relative gene expression data using real-time quantitative PCR and the 2(-Delta Delta C(T)) method. Methods 25: 402-408, 2001.

15. Zhang $X$, Lin $C$, Lu A, Lin G, Chen H, Liu Q, Yang Z and Zhang $\mathrm{H}$ : Liposomes equipped with cell penetrating peptide BR2 enhances chemotherapeutic effects of cantharidin against hepatocellular carcinoma. Drug Deliv 24: 986-998, 2017.

16. Li W, Xie L, Chen Z, Zhu Y, Sun Y, Miao Y, Xu Z and Han X: Cantharidin, a potent and selective PP2A inhibitor, induces an oxidative stress-independent growth inhibition of pancreatic cancer cells through $\mathrm{G} 2 / \mathrm{M}$ cell-cycle arrest and apoptosis. Cancer Sci 101: 1226-1233, 2010.

17. Garajová I, Ferracin M, Porcellini E, Palloni A, Abbati F, Biasco G and Brandi G: Non-Coding RNAs as predictive biomarkers to current treatment in metastatic colorectal cancer. Int J Mol Sci 18: pii: E1547, 2017.

18. van Beijnum JR, Giovannetti E, Poel D, Nowak-Sliwinska P and Griffioen AW: miRNAs: Micro-managers of anticancer combination therapies. Angiogenesis 20: 269-285, 2017. 
19. Deng J, Wang Y, Lei J, Lei W and Xiong JP: Insights into the involvement of noncoding RNAs in 5-fluorouracil drug resistance. Tumour Biol 39: 1010428317697553, 2017.

20. Mao XH, Chen M, Wang Y, Cui PG, Liu SB and Xu ZY: MicroRNA-21 regulates the ERK/NF- $\kappa \mathrm{B}$ signaling pathway to affect the proliferation, migration, and apoptosis of human melanoma A375 cells by targeting SPRY1, PDCD4, and PTEN. Mol Carcinog 56: 886-894, 2017.

21. Martin del Campo SE, Latchana N, Levine KM, Grignol VP, Fairchild ET, Jaime-Ramirez AC, Dao TV, Karpa VI, Carson M, Ganju A, et al: MiR-21 enhances melanoma invasiveness via inhibition of tissue inhibitor of metalloproteinases 3 expression: In vivo effects of MiR-21 inhibitor. PLoS One 10: e0115919, 2015.

22. Li Y, Wu R, Liu Z, Fan J and Yang H: Enforced expression of microRNA-21 influences the replication of varicella-zoster virus by triggering signal transducer and activator of transcription 3 . Exp Ther Med 7: 1291-1296, 2014.

23. Fu X, He Y, Wang X, Peng D, Chen X, Li X and Wang Q: Overexpression of miR-21 in stem cells improves ovarian structure and function in rats with chemotherapy-induced ovarian damage by targeting PDCD4 and PTEN to inhibit granulosa cell apoptosis. Stem Cell Res Ther 8: 187, 2017.

24. Bu LL, Yu GT, Wu L, Mao L, Deng WW, Liu JF, Kulkarni AB, Zhang WF, Zhang L and Sun ZJ: STAT3 Induces Immunosuppression by Upregulating PD-1/PD-L1 in HNSCC. J Dent Res 96: 1027-1034, 2017.
25. George S, Miao D, Demetri GD, Adeegbe D, Rodig SJ, Shukla S, Lipschitz M, Amin-Mansour A, Raut CP, Carter SL, et a: Loss of PTEN Is associated with resistance to anti-PD-1 checkpoint blockade therapy in metastatic uterine leiomyosarcoma. Immunity 46: 197-204, 2017.

26. Zhou K, Zhong Q, Wang YC, Xiong XY, Meng ZY, Zhao T, Zhu WY, Liao MF, Wu LR, Yang YR, et al: Regulatory T cells ameliorate intracerebral hemorrhage-induced inflammatory injury by modulating microglia/macrophage polarization through the IL-10/GSK3 $3 /$ PTEN axis. J Cereb Blood Flow Metab 37: 967-979, 2017.

27. Mondal S, Ghosh-Roy S, Loison F, Li Y, Jia Y, Harris C, Williams DA and Luo HR: PTEN negatively regulates engulfment of apoptotic cells by modulating activation of Rac GTPase. J Immunol 187: 5783-5794, 2011.

28. Conde-Perez A and Larue L: PTEN and melanomagenesis. Future Oncol 8: 1109-1120, 2012

29. Li WX, Chen X, Yang Y, Huang HM, Li HD, Huang C, Meng XM and Li J: Hesperitin derivative-11 suppress hepatic stellate cell activation and proliferation by targeting PTEN/AKT pathway. Toxicology 381: 75-86, 2017.

30. He Z, Chen AY, Rojanasakul Y, Rankin GO and Chen YC: Gallic acid, a phenolic compound, exerts anti-angiogenic effects via the PTEN/AKT/HIF-1 $\alpha /$ VEGF signaling pathway in ovarian cancer cells. Oncol Rep 35: 291-297, 2016.

31. Guo YB, Ji TF, Zhou HW and Yu JL: Effects of microRNA-21 on nerve cell regeneration and neural function recovery in diabetes mellitus combined with cerebral infarction rats by targeting PDCD4. Mol Neurobiol 55: 2494-2505, 2018. 